\title{
Impacto da depressão na condição bucal de pacientes portadores de síndrome metabólica: um estudo preliminar
}

\author{
Impact of depression in the oral condition of patients with metabolic syndrome: preliminary study \\ Impacto de la depresión en la condición oral de pacientes con síndrome metabólico: un estudio preliminar \\ Ana Carolina da Silva PINTO ${ }^{1}$ \\ ${ }^{1}$ Departamento de Odontopediatria, Ortodontia e Saúde Coletiva. Aréa de Saúde Coletiva. Faculdade de Odontologia de Bauru (FOB) \\ Universidade de São Paulo (USP) 17012-901 Bauru - SP, Brasil \\ ${ }^{2}$ Doutora em Ciências Odontológicas Aplicadas (Área de concentração: Saúde Coletiva) pela Faculdade de Odontologia de Bauru (FOB) \\ Universidade de São Paulo (USP) 17012-901 Bauru - SP, Brasil
}

\begin{abstract}
Resumo
A Síndrome metabólica (SM) é um conjunto de anormalidades metabólicas, antropométricas e hemodinâmicas. Avaliou-se cárie dentária e periodontite em obesos mórbidos com SM e depressão. Este estudo transversal e analítico foi composto por 56 pacientes divididos em 2 grupos: SM sem depressão (GSD=34) e SM com depressão (GCD =22). Utilizaram-se os índices ICDAS e CPOD para avaliar cárie dentária e o critério do CDC Periodontal Disease Surveillance Workgroup para periodontite. $\mathrm{Na}$ análise estatística utilizou-se: distribuição de Poisson, função Log de ligação e Regressão Logística Múltipla com distribuição binomial. Não houve diferença entre os grupos quanto à cárie dentária e periodontite. Conclui-se que pacientes com SM com ou sem depressão não apresentam diferenças significativas para cárie dentária e periodontite. Entretanto, pacientes com SM e depressão apresentaram maior perda dentária ( $p=0,0388)$. Novos estudos deverão investigar a relação entre SM, depressão e perda dentária.
\end{abstract}

Descritores: Síndrome X Metabólica; Periodontite; Cárie Dentária; Obesidade; Depressão.

\section{Abstract}

The metabolic syndrome (MS) is a set of metabolic, anthropometric and hemodynamic abnormalities. Dental caries and periodontitis were evaluated in morbidly obese with MS and depression. This transversal and analytic study was composed by 56 patients divided in two groups: MS without depression $(G S D=34)$ and MS with depression $(G C D=22)$. To evaluate dental caries, it was used the ICDAS and CPO-D indexes and to evaluate periodontitis it was used the criteria of the CDC Periodontal Disease Surveillance Workgroup. In the statistical analysis it was used: Poisson distribution, log link function and Multiple logistic regression with binomial distribution. There was no difference between the both groups regarding dental caries and periodontitis. It is concluded that patients with MS with or without depression do not have significant difference for dental caries and periodontitis. However, patients with MS and depression show higher tooth loss $(p=0,0388)$. New studies should investigate the relationship between MS, depression and tooth loss.

Descriptors: Metabolic Syndrome X; Periodontitis; Dental Caries; Obesity; Depression.

\section{Resumen}

El síndrome metabólico (SM) es un conjunto de anomalías metabólicas, antropométricas y hemodinámicas. Se evaluaron la caries dental y la periodontitis en obesos mórbidos con SM y depresión. Este estudio transversal y analítico incluyó 56 pacientes divididos en dos grupos: SM sin depresión (GSD = 34) y SM con depresión (MCD = 22). Se utilizaron los índices ICDAS y CPOD para evaluar la caries dental y los criterios del CDC Periodontal Disease Surveillance Workgroup para la periodontitis. En el análisis estadístico se utilizaron: distribución de Poisson, función de enlace log y regresión logística múltiple con distribución binomial. No hubo diferencias entre los grupos con respecto a la caries dental y la periodontitis. Se concluye que los pacientes con o sin depresión no tienen diferencias significativas en la caries dental y la periodontitis. Sin embargo, los pacientes con SM y depresión tuvieron una mayor pérdida de dientes $(p=0.0388)$. Otros estudios deberían investigar la relación entre la SM, la depresión y la pérdida de dientes.

Descriptores: Síndrome X Metabólico; Periodontitis; Caries Dental; Obesidad; Depresión.

INTRODUÇÃO

A síndrome metabólica (SM) consiste em um conjunto de anormalidades metabólicas, antropométricas e hemodinâmicas ${ }^{1}$ tais como hiperglicemia, hipertensão arterial, obesidade central e dislipidemia. Dentre todos os componentes da SM, o destaque é para a obesidade central, uma vez que se constitui no elemento predominante no contexto da síndrome $^{2-4}$. O diagnóstico da síndrome metabólica é confirmado quando o paciente possui obesidade central acrescida de pelo menos dois outros fatores, como: níveis elevados de triglicerídeos, redução de HDLColesterol, elevação da pressão arterial e elevação da glicemia de jejum ou diabetes mellitus tipo $2^{5}$.

Vale ressaltar que a associação entre alguns componentes da SM - especialmente 0 diabetes - e certos problemas bucais, tem sido bem documentada. Ademais, a SM está associada a um maior risco para transtornos mentais, 0 que parece ser devido, principalmente, à forte associação entre obesidade central e psicopatologias ${ }^{6}$.

Depressão e ansiedade são doenças comuns no mundo todo, com uma estimativa de 350 milhões de pessoas afetadas ${ }^{7}$. Há evidencias cientificas que demonstram uma associação entre doença mental e fatores comportamentais e fisiológicos. Fato este que sugere a possibilidade de a doença mental ser precursora de cárie dentária e doença periodontal $^{8}$. Dessa forma, saúde bucal e saúde sistêmica se encontram intimamente relacionadas, possivelmente, em razão do fato de que as doenças bucais e as doenças 
crônicas não transmissíveis têm fatores de risco comuns ${ }^{9}$.

No estudo de Timonen et al. ${ }^{10}$ achou-se uma associação, embora fraca, entre síndrome metabólica ou alguns de seus componentes e periodontite, sendo a resistência à insulina e a obesidade central os mais fortes determinantes em tal associação. Além disso, todos os componentes da síndrome, exceto dislipidemia, foram positivamente associados com cárie dentária.

É sabido que há necessidade de investigações que possam comprovar o papel da Síndrome Metabólica como determinante de doença periodontal e cárie dentária em pacientes com depressão. Levando em conta o aumento da ocorrência de depressão, SM, cárie dentária e doença periodontal, em adultos, torna-se relevante compreender melhor as relações entre estes distúrbios, para que se possa oferecer melhorias na sua prevenção e tratamento, bem como reforçar a importância da abordagem multiprofissional em face dos portadores.

MATERIAL E MÉTODO

- Aspectos éticos

Este trabalho foi realizado após avaliação e anuência do Comitê de Ética em Pesquisa em Seres Humanos do Hospital Amaral Carvalho (HAC), localizado no município de Jaú (SP), com aprovação ํㅜ 042675/2015. Foram seguidas as orientações de acordo com a Declaração de Helsinki.

- Tipo de estudo

O presente trabalho propôs a realização de um estudo do tipo transversal, a partir de dados secundários obtidos por meio de prontuários médico/odontológicos.

\section{- Composição da amostra}

A amostra foi composta por prontuários de pacientes obesos mórbidos candidatos à cirurgia bariátrica, com diagnóstico de Síndrome Metabólica (SM), podendo apresentar ou não depressão, que residiam em municípios pertencentes ao DRS VI - Bauru, atendidos no Hospital Amaral Carvalho, Jaú (SP). Este trabalho se propôs a analisar as condições bucais de pacientes obesos com Síndrome Metabólica, os quais foram divididos em dois grupos: $G C D=$ obesos com $S M$ e com depressão e $G S D=$ obesos com $S M$ e sem depressão.

\section{- Critérios de inclusão e exclusão}

Foram incluídos na amostra os pacientes obesos mórbidos candidatos à cirurgia bariátrica que apresentaram as seguintes condições:

- Diagnóstico de Síndrome Metabólica;
- Avaliação psicológica, realizada por membro da área de psicologia que integrava a equipe interdisciplinar do HAC, envolvendo aplicação de instrumento específico para classificação de sinais e sintomas depressivos.

Foram excluídos da amostra:

- Prontuários incompletos (ausência de dados necessários para a realização desta pesquisa) e ilegíveis;

- Pacientes com menos de 6 dentes.

- Coleta de dados

A coleta de dados foi realizada por meio dos prontuários de pacientes obesos candidatos à cirurgia bariátrica atendidos no $\mathrm{HAC}$ e na FOB-USP. O período de coleta de dados se deu entre junho de 2013 a maio de 2014.

\section{- Delineamento do estudo}

Foram randomizados 129 prontuários de pacientes obesos com indicação para cirurgia bariátrica, os quais se encontram arquivados no Ambulatório de Gastrenterologia e Cirurgia Bariátrica do HAC-Jaú (SP). Tais prontuários foram revisados buscando identificar, a princípio, a presença de Síndrome Metabólica (SM) e, na sequência, a condição psicológica, com relação ao grau da intensidade da depressão.

A medida da intensidade da depressão foi avaliada por uma psicóloga (E.C.G.) que integra a equipe interdisciplinar do HAC. O instrumento utilizado para tal mensuração foi o Inventário de Depressão de Beck (BDI) - versão $2001^{11}$, o qual consiste em uma escala autoadministrada, composta de 21 questões, cada uma com quatro alternativas, sugestionando graus crescentes de depressão. Com a totalização dos pontos é possível classificar o nível de intensidade da depressão dos pacientes que pode ser mínima (0-11), leve (12-19), moderada (20-35) e grave (36 a 63).

Para este estudo, foram considerados portadores de depressão os pacientes que apresentaram classificação correspondente aos níveis de depressão moderada e grave e os que faziam uso de medicamento para esta comorbidade.

\section{- Condições bucais}

\section{$\checkmark$ Cárie dentária}

Foram coletadas as informações referentes à cárie dentária utilizando os índices:

- CPOD: indica o número de dentes permanentes cariados, perdidos e restaurados.

- ICDAS: consiste num sistema de avaliação clínica padronizada que permite a detecção, avaliação, diagnóstico e 
monitorização de lesões de cárie dentária, tendo por base a atribuição de um código de dois dígitos a cada face dentária avaliada, no qual o primeiro se refere à presença ou ausência de restaurações ou selantes de fissuras e o segundo se refere à presença ou ausência de lesões de cárie.

\section{$\checkmark$ Doença periodontal}

Para avaliação da condição periodontal foi realizada a sondagem de seis sítios por dente (distovestibular, centro da face vestibular, mesiovestibular, distolingual, centro da face lingual e mesiolingual), em todos os dentes presentes na boca, utilizando, para tanto, a sonda periodontal tipo Carolina do Norte.

- Profundidade de sondagem: distância da margem gengival ao ponto mais apical do fundo do sulco/bolsa.

- Índice de sangramento gengival (ISG): presença ou ausência de sangramento após a sondagem do sulco gengival. É considerado ISG positivo quando ocorrer sangramento em até 10 segundos após a sondagem. $O$ número de achados positivos é expresso em percentagem relativo ao número de dentes presentes.

- Recessão gengival: distância, em milímetros, da junção cemento-esmalte até a altura da margem gengival nos seis sítios correspondentes à sondagem.

Neste trabalho, para identificar a presença de doença periodontal, adotou-se o critério proposto pelo CDC Periodontal Disease Surveillance Workgroup para definição de periodontite moderada, ou seja, foram considerados portadores de doença periodontal os pacientes que apresentaram recessão gengival interproximal de $4 \mathrm{~mm}$ ou mais em dois ou mais sítios interproximais, em dentes diferentes ou profundidade de bolsa periodontal de $5 \mathrm{~mm}$ ou mais em dois ou mais sítios interproximais, em dentes diferentes ${ }^{12}$.

\section{- Análise estatística}

Foi utilizado o programa SPSS versão 25 , para a realização das análises estatísticas. $\mathrm{O}$ teste Kolmogorov-Smirnov foi utilizado para investigar a hipótese de distribuição normal, em cada grupo, sendo esta não normal. Os testes adotados foram para dados não paramétricos. Foram realizadas as seguintes análises de regressão múltiplas: com distribuição de Poisson e função Log de ligação com as variáveis dependentes CPOD e ICDAS; Regressão Logística Múltipla com distribuição binomial e função Log de ligação com as variáveis dependentes "Presença de gengivite", "Presença de cálculo", "Presença de periodontite"; com distribuição e NIP. Adotou-se o nível de significância de $5 \%$ em todos os testes $(p<0,05)$

RESULTADOS

Foram analisados 129 prontuários, destes $57(60,6 \%)$ eram de portadores da Síndrome Metabólica. Após a aplicação dos critérios de elegibilidade restaram 56 prontuários. A média de idade destes pacientes foi de $38 \pm 9$ anos e $85,7 \%$ eram mulheres e $14,3 \%$ homens. Dentre os pacientes selecionados, 22 (39,2\%), apresentavam características de depressão, como mostrado na Tabela 1.

Entre as comorbidades estudadas, a glicemia de jejum estava presente em $50 \%$ no grupo GSD e 63,6\% no GCD. Em relação aos triglicerídeos no grupo GSD $73,5 \%$ e no GCD $45,45 \%$ tinham seus níveis elevados e em GSD $52,9 \%$ estavam com colesterol acima do nível de normalidade, já em GCD 36,36\%. Com relação à pressão arterial (PA) no grupo GSD $76,47 \%$ faziam uso de medicamento para o controle dessa morbidade e em GCD 95,45\%.

Tabela 1. Características sociodemográficas e distribuição de comorbidades (hipertensão, colesterol, diabetes, depressão)

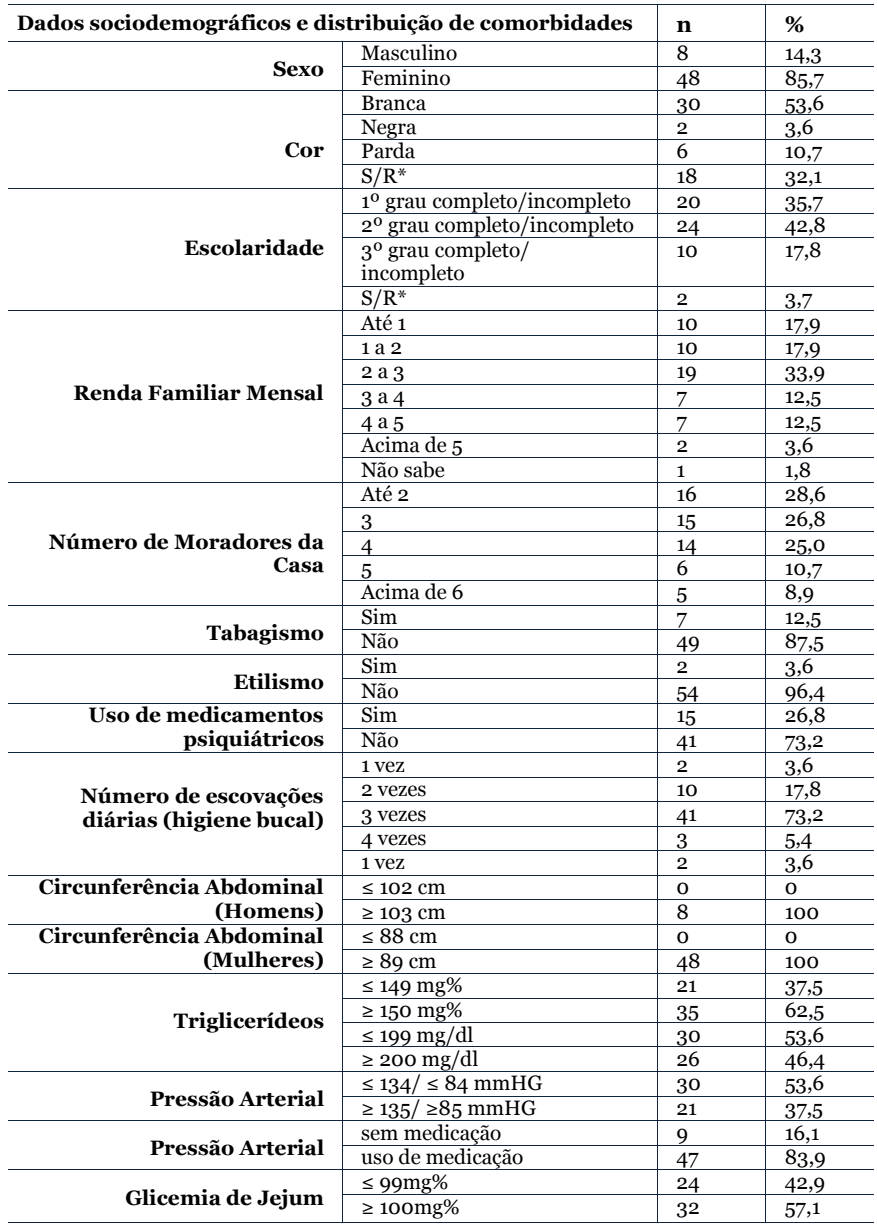

No grupo $G S D=41,17 \%$ dos pacientes apresentaram algum dente cariado, já no $\mathrm{GCD}=68,18 \%$ dos pacientes apresentaram 
cáries segundo o índice CPOD. A média de dentes perdidos no GSD foi de 2,73 dentes e no GCD essa média foi de 5,72, no grupo GSD $61,76 \%$ dos pacientes tiveram pelo menos um dente perdido por cárie, já no GCD $81,8 \%$. Já a média de dentes cariados segundo o CPOD no GSD foi de 13,14 e no GCD foi de 15,41 . Pelo índice ICDAS II a média de dentes cariados em GSD foi de 6,86 e em GCD 5,36, em relação ao número de pacientes com algum dente acometido por lesão cariosa em GSD 82,35\% de seus pacientes tiveram algum dente com lesão ativa, já em GCD 95,4\% dos pacientes possuiam lesão de cárie.

Em relação à cárie dentária em esmalte não houve diferenças entres os grupos $(p=0,557)$ e em dentina $(p=0,517)$. Já a análise do número de dentes perdidos mostrou que os pacientes de GCD apresentaram um número maior quando comparados ao grupo GSD, sendo estatisticamente significante $(p=0,0388)$, como se vê na Tabela 2.

Tabela 2. Comparação do perfil de cárie dentária entre os grupos com e sem depressão.

\begin{tabular}{|c|c|c|c|}
\hline Variáveis & $\begin{array}{l}\text { GSD } \\
\text { (Mediana) }\end{array}$ & $\begin{array}{l}\text { GCD } \\
\text { (Mediana) }\end{array}$ & Valor de $\mathbf{p}^{*}$ \\
\hline CPOD & 15 & 17 & 0,1372 \\
\hline Cariados & o & 1 & 0,1011 \\
\hline Perdidos & 1 & 3,5 & o,o388* \\
\hline Obturados & 8 & 7,5 & 0,4961 \\
\hline ICDAS médio*** & 0,225 & 0,710 & 0,1713 \\
\hline Hígido & 12,5 & 11,0 & 0,4448 \\
\hline Lesão inicial & 2 & 3 & 0,6006 \\
\hline Lesão cavitada & 0,00 & 1,00 & 0,1402 \\
\hline
\end{tabular}

$$
\text { No GSD, 59\% apresentaram }
$$

profundidade de bolsa > 4-5mm e em GCD $47 \%$. Em relação ao sangramento a sondagem no grupo GSD apresentou em média 2,5 dentes com sangramento e o grupo GCD 4,19. Quanto à profundidade de sondagem os grupos não apresentaram diferenças estatísticas $(p=0,273)$, nem em relação à recessão vestibular $(p=0,358)$, SG $(p=0,159)$ e NPI $(P=0,205)$. Quanto ao nível de inserção periodontal no GSD $77,2 \%$ dos pacientes tiveram esse índice superior a $4 \mathrm{~mm}$ e no GCD 67,6\%, como mostrado na Tabela 3.

Tabela 3. Análise de associação bivariada entre Depressão com gengivite, cálculo dentário e Doença periodontal

\begin{tabular}{|c|c|c|c|c|c|c|}
\hline \multirow{2}{*}{\multicolumn{2}{|c|}{ Variáveis de Desfecho }} & \multicolumn{2}{|c|}{ Sem Depressão } & \multicolumn{2}{|c|}{ Com Depressão } & \multirow[t]{2}{*}{ Valor de $\mathrm{p}^{*}$} \\
\hline & & $\mathrm{n}$ & $\%$ & $\mathrm{n}$ & $\%$ & \\
\hline \multirow{2}{*}{ Gengivite } & Não & 08 & 72,7 & 3 & 27,3 & \multirow{2}{*}{0,3671} \\
\hline & Sim & 26 & 57,8 & 19 & 42,2 & \\
\hline \multirow{2}{*}{ Cálculo } & Não & 11 & 68,8 & 5 & 31,2 & \multirow{2}{*}{0,4403} \\
\hline & Sim & 23 & 57,5 & 17 & 42,5 & \\
\hline \multirow{2}{*}{ Periodontite } & Não & 28 & 65,1 & 15 & 34,9 & \multirow{2}{*}{0,2241} \\
\hline & Sim & 6 & 46,2 & 7 & 53,8 & \\
\hline
\end{tabular}

DISCUSSÃO

Os resultados do presente estudo evidenciaram que indivíduos obesos com SM, com ou sem depressão, não apresentaram diferenças significativas para os desfechos cárie dentária e doença periodontal. Por outro lado, pacientes com SM e depressão apresentaram maior perda dentária. Estudos epidemiológicos e meta-análises já confirmaram a associação entre depressão e obesidade. Os mecanismos que associam essas duas condições têm sido muito estudados e vem indicando que o envolvimento da desregulação do eixo hipotálamo-pituitária-adrenal (HPA), inflamação, estresse oxidativo, bem como disfunção endócrina, podem ser responsáveis pela associação destas doenças ${ }^{13}$.

Neste estudo, 28,7\% dos participantes apresentaram glicemia de jejum superior ou igual a $110 \mathrm{mg}$, ou seja, se encontravam em risco de desenvolver diabetes mellitus, em GCD $50 \%$ e em GSD 35,3\%. A progressão da resistência à insulina e obesidade para T2DM continua a ser mal compreendida, mas implica uma falha de células $\beta$-pancreáticas para compensar a resistência à insulina, levando à hiperglicemia crônica. Inflamação crônica de baixo grau e ativação do sistema imunológico são observadas na obesidade abdominal e podem ter papel na patogênese da obesidade e de distúrbios metabólicos ${ }^{14}$.

Todos os pacientes desta pesquisa, tanto homens quanto mulheres apresentavam obesidade abdominal, considerada o melhor indicador para definir a presença da síndrome metabólica. Apesar disso, dos 129 prontuários de pacientes analisados, apenas 56 pacientes $(59,6 \%)$, foram classificados como portadores desse distúrbio. Esse número pode ser explicado pelo fato de que os dados utilizados para essa classificação eram referentes aos exames pré-operatórios dos pacientes. É sabido que, para a realização da cirurgia bariátrica, essas comorbidades devem estar, de certa forma, controladas. Portanto, é possível que, na população de obesos mórbidos que chegam ao serviço de cirurgia bariátrica, essa frequência seja ainda mais alta.

Os pacientes obesos são caracterizados por dois estados de resistência: resistência à insulina e a resistência à leptina, que estão fortemente associadas ao aumento da prevalência de doença periodontal e gengivite, assim como o risco aumentado de incidência de cárie dentária. Alguns parâmetros adicionais devem ser incluídos, tais como: os hábitos alimentares e fatores socioeconômicos e demográficos ${ }^{15}$.

$\mathrm{Na}$ amostra estudada, o grau de escolaridade foi um dado importante, uma vez que quanto maior o grau de conhecimento, melhores foram as práticas de saúde adotadas. Foi possível observar que a maioria dos pacientes apresentou baixa escolaridade, não 
tendo completado o segundo grau. Um estudo relatou que sujeitos com baixo nível de escolaridade apresentavam maior número de dentes ausentes do que aqueles com alto nível de escolaridade ${ }^{16}$. Observou-se no nosso estudo que a maioria das famílias apresentava como renda até três salários mínimos, o que pode, também, influenciar nos hábitos de higiene bucal, já que muitas vezes não é possível o acesso ao dentifrício, fio dental ou mesmo escovas dentais para toda a família. Estudos mostram que a diminuição do status socioeconômico está associada ao desenvolvimento de cárie ${ }^{17}$. Porém, ainda assim, a maioria dos pacientes afirmou escovar os dentes três vezes ao dia, o que talvez se explique pelo fato de 0 paciente convenientemente não querer assumir e assinar um atestado de desleixo e culpabilidade pelo próprio mau estado bucal.

A literatura científica apresenta a relação entre obesidade e problemas bucais, tais como redução do fluxo salivar, desgaste dentário, carie, doença periodontal e perda dentária ${ }^{18,19}$. Já a depressão esteve fortemente associada à cárie dentária, em um estudo que controlou as variáveis de confusão, características sociodemográficas, doenças sistêmicas, comportamentos ligados à saúde e o uso de medicamentos ${ }^{8}$.

No presente estudo, relacionou-se depressão, SM e condições bucais. Cárie e SM compartilham fatores de risco comuns, tais como: o consumo de álcool, tabagismo, baixa atividade física e dieta rica em carboidratos. A National Health and Nutrition Examination Survey mostrou que adultos com idade $\geq 20$ anos, com a doença cárie tinham maiores chances de ter o IMC $\geq 30.0 \mathrm{~kg} / \mathrm{m} 2$ do que aqueles que não tinham a doença. A relação entre cárie e obesidade também foi associada à redução do fluxo salivar, já que a obesidade desencadeia a hipofunção da glândula salivar e a hiposalivação aumenta a susceptibilidade a cáries $^{20}$. Apesar disso, não há consenso na literatura em relação à associação entre obesidade e cárie dentária.

Foi possível observar alta prevalência de cárie nos pacientes analisados, sendo que em sua maioria apresentaram história da doença. No presente estudo, a cárie dentária foi avaliada adotando-se dois índices epidemiológicos (ICDAS e CPOD). No primeiro índice podem-se detectar as lesões iniciais, já no segundo podem-se mensurar as lesões cavitadas em dentina. Entre os pacientes avaliados em $\mathrm{GCD}=68,18 \%$ apresentaram algum dente cariado, já no $\mathrm{GSD}=41,17 \%$ apresentaram cáries segundo o índice CPOD. A média de dentes perdidos no GCD foi de 5,72 dentes e no GSD essa média foi de 2,73. Já a média de dentes cariados segundo o CPOD no GCD foi de 15,41 e no GSD foi de 13,14. Pelo índice ICDAS II a média de dentes cariados em GCD foi de 6,86 e em GSD de 5,36 e em relação ao número de pacientes com algum dente acometido por lesão cariosa o GCD teve $95,4 \%$ de seus pacientes com alguma lesão ativa, já em GSD 82,35\% dos pacientes possuía lesão de cárie.

Os pacientes de GCD tiveram um maior número de dentes perdidos quando comparados com o grupo GSD $(p=0,0388)$. Estudos sugerem que as disparidades na prevalência do uso de serviços de saúde bucal e a perda dentária existem entre pessoas com depressão e ansiedade, além disso, os adultos sem diagnóstico de depressão e ansiedade tiveram uma prevalência significativamente maior de não ter nenhum dente removido devido à cárie dentária ou doença periodontal ${ }^{21}$.

A avaliação da condição periodontal demonstrou que os pacientes avaliados apresentavam diferentes graus de severidade da doença periodontal. Podemos observar que no grupo $\mathrm{GCD}=59 \%$ apresentaram profundidade de bolsa $>4-5 \mathrm{~mm}$ e em GSD= $47 \%$. Relativamente ao sangramento a sondagem no grupo GCD apresentou em média 4,19 dentes com sangramento e GSD 2,5. Deve-se considerar que os fatores de exposição para depressão e SM, tais como uso de medicamentos, hábitos de higiene e alimentares inadequados, podem contribuir para o desencadeamento das doenças cárie dentária e periodontal. A doença psiquiátrica pode levar a uma má saúde bucal por causa do estilo de vida, má higiene bucal e dificuldades no acesso aos cuidados dentários ${ }^{22,23}$.

As explicações para o aumento dos níveis de cárie incluem a higiene bucal deficiente $e$ os efeitos colaterais dos medicamentos psicotrópicos, como antipsicóticos, antidepressivos e estabilizadores do humor. Todos esses medicamentos induzem a boca seca (xerostomia) através da redução do fluxo salivar e podem potencialmente negar os efeitos benéficos do flúor ${ }^{24}$. Tal como acontece com outros aspectos da má saúde física, a má saúde dental também pode estar relacionada à má alimentação, tabagismo e má higiene bucal $^{25}$.

Uma limitação deste estudo foi que não consideramos, para a classificação da SM, os dados de HDL-colesterol, uma vez que apenas informações referentes a colesterol total 
encontravam-se disponíveis. Este dado foi coletado e utilizado para a classificação da SM por meio de uma adaptação da proposta apresentada pelo NCEP ATP III, sendo considerado, como parâmetro, níveis superiores a $200 \mathrm{mg} / \mathrm{dl}$, que representa, de acordo com a Organização Mundial de Saúde (OMS), o ponto de corte acima do qual um indivíduo se encontra sob risco de doença cardiovascular ${ }^{26}$. Cerca de $30 \%$ dos pacientes diagnosticados com a síndrome metabólica apresentaram níveis de colesterol total acima do recomendado pela OMS e no grupo GCD 40,9\% estavam com ele acima do nível de normalidade e em GSD 50\%. Outro ponto a ser discutido é o tipo de estudo, transversal, o qual não permite fazer a relação causa-efeito. Estudos futuros deverão ser conduzidos com amostras maiores e por longo período de seguimento para que as relações causais possam ser esclarecidas.

Apesar da alta prevalência de cárie dentária e doença periodontal, a análise de associação bivariada entre depressão e componentes da SM com essas variáveis não apresentaram diferenças significativas. Entretanto, pacientes portadores de SM e depressão necessitam de acompanhamento odontológico. A relação entre condições bucais e depressão em pacientes portadores de síndrome metabólica deve ser investigada em outros estudos para se esclarecer esta questão.

\section{CONCLUSÃO}

Com os resultados apresentados neste trabalho pode-se concluir que não houve associação entre depressão e as doenças cárie dentária e periodontal em pacientes com SM. Entretanto, os pacientes com SM e depressão apresentaram maior perda dentária.

AGRADECIMENTOS

Os autores agradecem à Fundação de Amparo à Pesquisa do Estado de São Paulo (FAPESP- 14/13315-2) e à Coordenação de Aperfeiçoamento de Pessoal de Nível Superior Brasil (CAPES - 001), por apoiar este estudo.

\section{REFERÊNCIAS}

1. Toker S, Shirom A, Melamed S. Depression and the metabolic syndrome: genderdependent associations. Depress Anxiety. 2008;25(8):661-69.

2. Gagliardi AC, Miname MH, Santos RD. Uric acid: A marker of increased cardiovascular risk. Atherosclerosis. 2009;202(1):11-7.

3. Eckel RH, Grundy SM, Zimmet PZ. The metabolic syndrome. Lancet. 2005; 365(9468):1415-28.

4. Després JP, Lemieux I. Abdominal obesity and metabolic syndrome. Nature. 2006;444:881-7.
5. International Diabetes Federation (IDF) The IDF consensus worldwide definition of the metabolic syndrome 2006. Disponível em: < http://www. idf.org/webdata/docs/IDF_Meta_def_final.pdf

6. Carpiniello B, Pinna F, Velluzzi F, Loviselli A. Mental disorders in patients with metabolic syndrome. The key role of central obesity. Eat Weight Disord. 2012;17(4):e259-66.

7. World Health Organization, World suicide prevention day 2012. http://www.who.int/ mediacentre/events/annual/world_suicide_prev ention_day/en/

8. Delgado-Angulo EK, Sabbah W, Suominen AL, Vehkalahti MM, Knuuttila $M$, Partonen $T$, Nordblad A, Sheiham A, Watt RG, Tsakos G. The association of depression and anxiety with dental caries and periodontal disease among Finnish adults. Community Dent Oral Epidemiol. 2015;43(6):540-49.

9. Petersen PE, Ogawa $H$. Strengthening the prevention of periodontal disease: the $\mathrm{WHO}$ approach. J Periodontol. 2005;76(12):2187-93.

10. Timonen $P$, Niskanen M, Suominen-Taipale L, Jula A, Knuuttila M, Ylöstalo P. Metabolic syndrome, periodontal infection, and dental caries. J Dent Res. 2010;89(10):1068-73.

11. Cunha JA. Manual da versão em português das escalas Beck. São Paulo, SP: Casa do Psicólogo; 2001.

12. Eke PI, Genco RJ. CDC Periodontal Disease Surveillance Project: background, objectives, and progress report. J Periodontol. 2007; 78(7 Suppl):1366-71.

13. Jantaratnotai N, Mosikanon K, Lee $\mathrm{Y}$, McIntyre RS. The interface of depression and obesity. Obes Res Clin Pract. 2017;11(1):1-10

14. Paquot $N$. de la médecine factuelle à la médecine personnaliée: l'exemple du diabète de type 2 [From evidence-based medicine to personalized medicine: the exemple of type 2 diabetes]. Rev Med Liege. 2015;70(5-6):299305.

15. Słotwińska SM, Słotwiński R. Host response, obesity, and oral health. Cent Eur J Immunol. 2015;40(2):201-5.

16. Ueno $M$, Ohara $S$, Inoue $M$, Tsugane $S$, Kawaguchi $\mathrm{Y}$. Association between education level and dentition status in Japanese adults: Japan public health center-based oral health study. Community Dent Oral Epidemiol. 2012; 40(6):481-87.

17. Gerdin EW, Angbratt M, Aronsson K, Eriksson E, Johansson I. Dental caries and body mass index by socio-economic status in Swedish children. Community Dent Oral Epidemiol. 2008;36(5):459-65.

18. Moura-Grec PG, Assis VH, Cannabrava VP, Vieira VM, Siqueira TL, Anaguizawa WH, Sales-Peres SH. Systemic consequences of bariatric surgery and its repercussions on oral health. Arq Bras Cir Dig. 2012;25(3):173-77. 
19. Yamashita JM, Moura-Grec PG, Freitas AR, Sales-Peres A, Groppo FC, Ceneviva R et al. Assessment of Oral Conditions and Quality of Life in Morbid Obese and Normal Weight Individuals: A Cross-Sectional Study. PloS one.2015;10(7):e0129687.

20. Ojima M, Amano A, Kurata S. Relationship between decayed teeth and metabolic syndrome: data from 4716 middle-aged male Japanese employees. J Epidemiol. 2015; 25(3):204-11.

21. Okoro CA, Zhao G, Li C, Balluz LS. Use of complementary and alternative medicine among US adults with and without functional limitations. Disabil Rehabil. 2012;34(2):128-35.

22. Kisely S, Baghaie $H$, Lalloo $R$, Siskind D, Johnson NW. A systematic review and metaanalysis of the association between poor oral health and severe mental illness. Psychosom Med. 2015;77(1):83-92.

23. Kisely S, Sawyer E, Siskind D, Lalloo R. The oral health of people with anxiety and depressive disorders - a systematic review and meta-analysis. J Affect Disord. 2016;200: 119-32.

24. Friedlander $\mathrm{AH}, \quad$ Marder SR. The psychopathology, medical management and dental implications of schizophrenia. J Am Dent Assoc. 2002;133(5):603-10; quiz 624-5.

25. McCreadie RG, Stevens H, Henderson J, Hall D, McCaul R, Filik R, Young G, Sutch G, Kanagaratnam G, Perrington S, McKendrick J, Stephenson D, Burns T. The dental health of people with schizophrenia. Acta Psychiatr Scand. 2004;110(4):306-10.

26. Capewell S, Ford ES, Croft JB, Critchley JA, Greenlund KJ, Labarthe DR. Cardiovascular risk factor trends and potential for reducing coronary heart disease mortality in the United States of America. Bull World Health Organ. 2010;88(2):120-30.

\section{CONFLITO DE INTERESSES}

Os autores declaram não haver conflitos de interesse

\section{AUTOR PARA CORRESPONDÊNCIA}

\section{Ana Carolina da Silva Pinto}

Departamento de Ortodontia, Odontopediatra e Saúde Coletiva

Faculdade de Odontologia de Bauru (FOB)

Universidade de São Paulo (USP)

17012-901 Bauru - SP, Brasil

E-mail: ana.pin@usp.br 